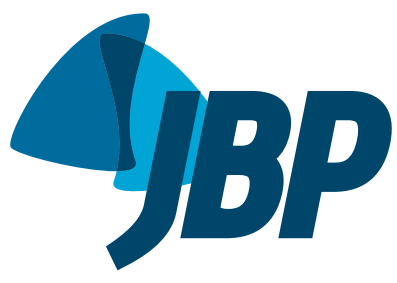

\title{
The rapid shallow breathing index as a predictor of successful mechanical ventilation weaning: clinical utility when calculated from ventilator data
}

\author{
Luiz Alberto Forgiarini Junior ${ }^{1}$, Antonio M. Esquinas ${ }^{2}$
}

First, we would like to congratulate Souza et al. for their article entitled "The rapid shallow breathing index as a predictor of successful mechanical ventilation weaning: clinical utility when calculated from ventilator data", recently published in the JBP. ${ }^{(1)}$ The subject is of great importance for professionals in the intensive care unit, and the article presents a new tool for the evaluation of critically ill patients on mechanical ventilation.

In the literature, there are a variety of studies demonstrating the use of rapid shallow breathing index (RSBI) calculated directly from the mechanical ventilator data. However, the poor methodological quality of those studies makes it impossible to implement the RSBI directly in clinical practice or to determine the appropriate cut-off point for the index. The development of new assessment tools that can be adapted for use in daily practice is extremely important to the efficiency of care provided by a multidisciplinary team. Improvements in the use of predictive factors for weaning have been reported in the literature. For example, Takaki et al. (2) conducted a study designed to evaluate the best predictors of successful extubation in the cardiac intensive care unit by correcting the RSBI values for the anthropometric characteristics of the patients. Those authors demonstrated that the modified RSBI adjusted for the current body weight or body mass index has greater predictive power than does the conventional RSBI.
One interesting finding presented by Souza et al. ${ }^{(1)}$ is that even if there is significant difference in the direct comparison of the RSBI obtained by spirometry or directly from the ventilator, the two are very similar in terms of accuracy, which justifies the use of either method. Although we observed a statistically significant difference in the direct comparison of measurement methods, the values obtained in both groups were below 105, which is indicative of successful weaning.

We believe that research related to an index or tools for assessing weaning potential must also take into account the population studied. For example, the RSBI has not proven to be a reliable predictor of successful weaning in patients with neurological damage. This is clear in the study conducted by Kutchak et al.,(3) who evaluated the use of reflex cough PEF as a predictor of successful extubation in neurological patients. Those authors showed that, although all of the patients (those in whom extubation was successful and those in whom it was not) presented an RSBI < 105, reflex cough PEF had an accuracy of 0.81 in predicting successful extubation.

Certainly, despite the interesting findings of the Souza et al. (1) study, new research demonstrating the effectiveness of the RSBI in different populations or adjusting its values by clinical factors should be encouraged.

\section{REFERENCES}

1. Souza LC, Lugon JR. The rapid shallow breathing index as a predictor of successful mechanical ventilation weaning: clinical utility when calculated from ventilator data. J Bras Pneumol. 2015;41(6):530-5. http://dx.doi.org/10.1590/S1806-37132015000000077

2. Takaki S, Kadiman SB, Tahir SS, Ariff MH, Kurahashi K, Goto T. Modified rapid shallow breathing index adjusted with anthropometric parameters increases predictive power for extubation failure compared with the

unmodified index in postcardiac surgery patients. J Cardiothorac Vasc Anesth. 2015;29(1):64-8. http://dx.doi.org/10.1053/j.jvca.2014.06.022

3. KutchakFM, Debesaitys AM, Rieder Mde M, MeneguzziC, SkuereskyAS, Forgiarini Junior LA, et al. Reflex cough PEF as a predictor of successful extubation in neurological patients. J Bras Pneumol. 2015;41(4):358-64. http://dx.doi.org/10.1590/S1806-37132015000004453 